\section{Niklas Schrape}

\section{Die Rhetorik von Computerspielen}

Wie politische Spiele überzeugen

Frankfurt am Main: Campus , 2012. - 356 S.

ISBN: 978-3-593-39795-5

Zugl.: Potsdam, Hochschule für Film und

Fernsehen, Diss. 2011

Wie vermitteln Spiele Informationen und wie überzeugen sie die Nutzer von ihrer Relevanz? Dies sind nur zwei Fragen der stetig weiter ausdifferenzierten Forschung zu digitalen Spielen. Egal ob Spieler in der Mittagspause Figuren auf dem Smartphone rudimentäre Bewegungen ausführen lassen oder in der Freizeit ganze Unternehmen, Städte oder gar Welten managen meist werden die zu erfüllenden Aufgaben mit einem Kontext verknüpft, der nicht nur vorgegebene Handlungsmöglichkeiten legitimiert, sondern auch ihre Bedeutung betont.

Auch bei der vorliegenden Arbeit von Niklas Schrape stehen diese Fragen im Mittelpunkt. Als Ziel seiner Dissertation, die 2011 an der Hochschule für Film und Fernsehen in Potsdam angenommen wurde, erklärt er, einen „Ansatz einer medienspezifischen Rhetorik von Computerspielen entwickeln" (S.8) zu wollen, wozu er „Ähnlichkeiten wie Differenzen zwischen Computerspiel und rhetorischer Rede“ (S. 10) untersucht. Im ersten Drittel des Buches wird dazu eine Methodik zur Analyse der Spielrhetorik entwickelt, die dann im verbleibenden Teil an zwei Fallbeispielen überprüft wird: dem Strategiespiel „Peacemaker“ (PM, Impact Games 2007) und dem Adventure "Global Conflicts: Palestine" (GCP, Runesoft, Serious Games Interactive 2007), die beide den israelisch-palästinensischen Konflikt thematisieren.

Kern des Ansatzes von Schrape bildet die Annahme, dass "Computerspiele als Texte“ (S. 25) beschrieben werden können, wobei er sich besonders auf Aarseth und Bogost sowie zu geringeren Teilen auf Eskelinen und Frasca stützt. Andere Ansätze aus den Game Studies, wie von Juul, Neitzel, Nohr, Pias oder Salen/ Zimmerman, werden hingegen kaum berücksichtigt. Die Spielrhetorik ist vielmehr von Barthes, Kanzogs, Lotmans, Knillis und Reisse Beiträgen zur Literatur- und Filmwissenschaft aus den 1950er bis 70er Jahren sowie Caillois Ansätzen zur allgemeinen Spielforschung aus dem gleichen Zeitraum geprägt.

Zwar distanziert sich Schrape eingangs von frühen narratologischen Ansätzen der Spielforschung und hebt die Bedeutung des Systemischen beim Spielerleben hervor (S. 61), jedoch bleibt sein Modell, das im Kern aus dem $\mathrm{Zu}$ sammenspiel von innerer und äußerer Rhetorik sowie System und Interface besteht, insgesamt stark narratologisch geprägt. Auch der wiederkehrende Bezug auf die „Aufgaben des Redners nach Cicero“ und die „Mittel der Überzeugung nach Aristoteles" (S. 39) unterstreicht dies, weil damit impliziert wird, dass Spielentwickler an die Stelle des Redners treten, die Spieler mit ihren Werken argumentativ von bestimmten Themen (hier: die Lösung des Nahost-Konflikts) überzeugen wollen.

Da der Nahost-Konflikt durch die häufige Diskussion in den Medien bereits allgemein bekannt ist, können sowohl PM als auch GCP auf vielfältige Vorstellungen bei ihren Nutzern aufbauen. Während über Schrapes Modell damit beschrieben werden kann, wie Appelle, Aporien, Instruktionen und weitere Mittel zu einem bestimmten Verständnis der Spielinhalte anleiten, bleibt gerade in GCP ein den realen Friedensprozess hemmender Aspekt weitgehend ausgeklammert: der ideologische Hintergrund der jeweiligen Protagonisten und die Einbindung in ihr politisches Umfeld.

Eine Erläuterung der Gründe für diese Lücke, die bei Anforderungen an das Spieldesign (Gewinnbarkeit, verschiedene Vorgehensweisen etc.) zu suchen sind und einen wichtigen Unterschied zwischen realem und virtuellem Nahost-Konflikt markieren, wäre hilfreich gewesen, um die Rhetorik besser in den Game Studies verorten zu können. Während entsprechende weiterführende Erläuterungen in eigenen Worten generell Verständnis fördernd sind, beschränkt sich Schrape jedoch meist darauf, verschiedene Positionen aus der Literatur gegenüberzustellen. Die beiden enthaltenen Glossars zur Computerspiel- bzw. Bild- und Filmrhetorik sind ebenfalls nur bedingt hilfreich, weil darin in der Regel fachspezifische Termini mit weiteren umschrieben werden.

Im Fazit finden sich schließlich neben einer Zusammenfassung des Gesagten und einem Ausblick zur Zukunft des Themas auch längere Ausführungen zur Frage, ob „gewalthaltige Computerspiele Aggressionen befördern" (S. 314), man zielen lernen könne (S. 319) oder zum begrenzten Wert von computerbasierten Simulationsmodellen am Beispiel der aktuellen Finanzkrise und dem Reaktorunglück von Fukushima (S. 323). Der Bezug zum Thema der Arbeit bleibt dabei vage.

Schrapes Computerspielrhetorik liefert damit zusammengefasst zwar eine Reihe neuer Ansätze, die für die Analyse von Spielen hilfreich sein können, jedoch ist sie zu stark an der Literatur- und Filmwissenschaft orientiert und 
berücksichtigt Methoden der Game Studies zu wenig. Bedingt durch die Interaktivität des $\mathrm{Me}-$ diums können Entwickler zwar versuchen, von bestimmten Schlussfolgerungen zu überzeugen, jedoch bleibt es dem Spieler überlassen, ob er sich auf diese Versuche einlässt oder aber das Spiel nutzt, wie es ihm gefällt.

Lutz Schröder

\author{
Sabine Trepte / Leonard Reinecke \\ Medienpsychologie \\ Stuttgart: Kohlhammer, 2013. - 276 S. \\ (Grundriss der Psychologie; 27) \\ ISBN 978-3-17-021438-5
}

Als ich gefragt wurde, ob ich den Band „Medienpsychologie" von Sabine Trepte und Leonard Reinecke in der Reihe „Grundriss der Psychologie" rezensieren möchte, habe ich zugestimmt und ein weiteres dickes Lehrbuch der Medienpsychologie erwartet. Umso überraschter war ich, als das Buch dann ankam - eher ein Bändchen trotz der knapp 270 Seiten. Das handliche und kompakte Format weckt in jedem Fall die Leselust.

Für Leser, die mit der von Maria von Salisch und Bernd Leplow herausgegebenen Reihe noch nicht vertraut sind, macht das Geleitwort die primäre Zielgruppe deutlich: Bachelorstudenten. Die einzelnen Bände sollen unabhängig voneinander gelesen werden können und das Lehrgebiet des Bachelor-Studiums beschreiben. Dementsprechend ist das Buch auch gestaltet - in jedem Kapitel finden sich farblich abgesetzte Definitionen, Merksätze und am Ende jeweils Literaturempfehlungen und Fragen zur Selbstüberprüfung. Damit ist das Buch auf jeden Fall sehr studierendenfreundlich aufgebaut.

Inhaltlich gliedert es sich in Einleitung, Methoden der Medienpsychologie, Medienselektion, Medienrezeption, Medienwirkung, computervermittelte Kommunikation, MenschComputer-Interaktion und Medienkompetenz. Etwas überraschend schließt sich daran noch ein Kapitel zu den Berufsfeldern an.

Das einleitende Kapitel beginnt mit einer Definition der Medienpsychologie, ausgehend von den beiden Wortbestandteilen Medien und Psychologie. Verwirrenderweise fokussiert der Medien-Abschnitt zunächst nur auf Massenmedien. Hier wäre es hilfreicher gewesen, die grobe Unterscheidung in Massen- und Individualkommunikation erst anzukündigen, bevor ins Detail gegangen wird. Als mögliche Strukturierungsdimensionen werden dann die psy- chologische Trias von Emotion, Kognition und Verhalten sowie die Einteilung anhand der Formen der Mediennutzung (Selektion, Rezeption, Wirkung, medienvermittelte Kommunikation) vorgestellt. Der Oberbegriff „Formen“ ist nicht ganz glücklich gewählt, da Selektion, Rezeption und Wirkung sich auch auf die medienvermittelte Kommunikation anwenden ließen.

Das Methodenkapitel behandelt vor allem Experiment, Befragung und psychophysiologische Methoden. Das Experiment wird implizit favorisiert, da dazu nicht wie bei den anderen Methoden die Vor- und Nachteile aufgeführt werden. Auch in den weiteren Kapiteln werden immer wieder exemplarisch Experimente zum jeweiligen Thema näher beschrieben. Damit richtet sich der Band vor allem auf die quantitativen und klassischen Methoden der Psychologie; Inhaltsanalyse und qualitative Verfahren werden unter weitere Methoden abgehandelt.

Im Allgemeinen geben die Kapitel einen guten Überblick über die jeweiligen Themen. Die Darstellung ist kurz und prägnant. Im Kapitel zur Medienrezeption wird dicht gedrängt eine solche Fülle von Ansätzen vorgestellt, dass der Leser schon beinahe den Überblick verliert. Der Abschnitt über Unterhaltungserleben hätte vielleicht besser an den Schluss gestellt werden können, um mit einer eingehenderen Beschreibung des Model of Complex Entertainment Experiences stärker eine integrierende Klammer zu bieten.

Beim Thema Medienwirkung werden zunächst allgemeine theoretische Ansätze zur kognitiven und emotionalen Medienwirkung erläutert, bevor die Effekte auf aggressives und (verringertes) prosoziales Verhalten in einem separaten Kapitel behandelt werden. Auch hier zeigt sich, dass es schwierig ist, die Medienwirkungsforschung in einem begrenzten Band darzustellen. Der Fokus liegt auf Bereichen, die sich gut experimentell untersuchen lassen; Kultivierungseffekte werden dagegen nicht besprochen.

Ab Kapitel 7 wendet sich das Buch eher der Individualkommunikation zu. Das Kapitel zur computervermittelten Kommunikation weist jedoch einige Schwächen auf. Bei der Beschreibung der klassischen Modelle kommt der historische Kontext zu kurz. Die Entwicklung von anonymen/pseudonymen Formen der computervermittelten Kommunikation hin zur nonymen Kommunikation in sozialen Netzwerken und die Implikationen für die jeweiligen Modelle hätten deutlicher herausgearbeitet werden können. Der Social Information ProcessingPerspektive von Walther wird der Status als Modell oder Theorie abgesprochen, es werden 